\title{
The Black Civil Rights Movement in America from 1950s to 1960s
}

\author{
He Yufeng ${ }^{1, a}$, Zhu Rui ${ }^{2, b^{*}}$ \\ ${ }^{1}$ College of Political Science and Law, Capital Normal University, Haidian, Beijing, China \\ ${ }^{2}$ College of Political Science and Law, Capital Normal University, Haidian, Beijing, China \\ a2682423583@qq.com \\ b*2271400209@qq.com
}

\begin{abstract}
African Americans can't use the same public facilities with white people. But America is also advocating itself as a"Free land",contradicting with the existence racial segregation. Under the effects of various factors, African Americans started to fight for equal rights and freedom. This movement reached its zenith in 1950s-1960s and African Americans finally gained equality, liberty and dignity. But the racial discrimination is still a significant issue in America, which received a huge amount of attention after Freud was killed by white police. At the same time, African Americans chasing for equality excessively, which cause other social problems, such as political correctness issues and American society being torn up.
\end{abstract}

Keywords : Civil Rights Movement, African American, Political Correctness

\section{二十世纪五十至六十年代非裔美国人民权运动问题探 究}

何雨峰 ${ }^{1, a}$ 朱瑞 ${ }^{2,}$ b*

${ }^{1}$ 首都师范大学政法学院, 海定, 北京, 中国

2 首都师范大学政法学院, 海淀, 北京, 中国

a2682423583@qq.com

b*2271400209@qq.com

\section{摘要:}

非洲裔美国人无法与白人公用公共设施。但美国却在不断宣扬其是一个自由之地，但这却与种族隔离的存在格 格不入。在各种因素的作用下，非裔美国人开始不断争取自身权利的平等与自由，最终在五六十年代轰轰烈烈 的民权运动达到了最高峰，非裔美国人就此获得了很大程度上的平等、自由和尊严。但同时种族歧视问题依然 在美国国内是一个重大议题，自弗洛伊德事件之后，对于种族歧视的关注再度获得大量关注。同时非裔美国人 过于追求平等，也同样导致了美国社会出现了其他问题，比如政治正确问题与社会撕裂问题。

关键词：民权运动，非裔美国人，政治正确

\section{1. 前言}

黑人民权运动（Black Civil Rights Movement）, 是美国民权运动的一部分, 是非裔美国人族裔为了追 求与白人平等的地位而发起的一场运动。该运动在 1954-1968 年期间成功消除了美国的种族隔离制度,
非裔美国人就此获得了很大程度上的平等、自由和尊 严。但这项运动并不彻底, 至今日种族歧视问题依然 在美国国内是一个重大议题; 自弗洛伊德事件之后, 对于种族歧视的关注再度获得大量关注; 同时由于黑 人族裔没有明确的政治纲领，过度追求权利导致社会 言论被“政治正确”所把控，同样使得美国社会出现了 其他问题，比如过度的政治正确与社会撕裂问题。 


\section{2. 二十世纪五十至六十年代非裔美国人民权 运动发生的背景}

自 17 世纪早期开始, 伴随着北美殖民地的开拓 与对本土印第安人的迫害, 导致美洲大陆劳动力紧缺, 随后通过臭名昭著的“三角贸易”，欧洲殖民者一批批 地将从非洲捕获或者交易换来的黑人运往美洲。1661 年弗吉尼亚州通过一部法律, 确定了永久奴隶制, 简 而言之，黑人将世代为奴。自此之后，美洲各处都纷 纷援引这一先例。这些黑奴为了美国的资本主义发展 献上了自己的生命。同时, 黑奴也在人身自由与权利 上收到了极大限制, 他们是奴隶, 失去了作为一名美 国公民的权利, 虽然在美国南北战争之后, 黑人获得 了名义上的解放, 但种族歧视与种族隔离依然对于非 裔美国人是一个巨大的束缚。非洲裔美国人无法与白 人公用公共设施。除此之外, 在第一次世界大战之后, 即便黑人在美国军队当中表现卓著, 帮助协约国战胜 了同盟国, 但国内的白人依然使用暴力手段阻止他们 获得正当的权利。但美国却在不断宣扬其是一个自由 之地, 但这却与种族歧视的存在格格不入, 越来越多 的非裔美国人无法容忍这种理想与现实之间的巨大 鸿沟。随着第二次世界大战的爆发, 越来越多的非裔 美国人士兵受到了公平的对待, 与他们在国内的待遇 存在着巨大的差距, 而且联邦政府也在逐步出台政策, 改善他们的环境。第二次世界大战也使得非裔美国人 在社会经济方面获得了重大改善, 地位越来越重要, 给予了他们谋求改变的力量。自此, 越来越多的非裔 美国人已经无法容忍这种现实和理想之间的巨大差 距, 他们认识到只有在法律上彻底废除种族隔离这一 枷锁, 才有可能实现黑人族裔与白人族裔的彻底平等, 在这一思想的驱使之下, 大量非裔美国人开始走上街 头, 使用各种方式, 去破除种族主义与种族隔离给他 们带上的枷锁。除此之外, 全美也发起了自由主义 宪政革命, 不仅是黑人这一族裔, 许多白人族裔也加 入其中，帮助黑人族裔摆脱他们的困境。

\section{3. 二十世纪五十至六十年代非裔美国人民权 运动产生原因}

民权运动产生的原因并不仅仅只是长期的蓄奴 制经济与种族隔离, 事实上, 其中还有更多因素导致 了五六十年代轰轰烈烈的民权运动。原因可以被归结 为以下几点: 美国长期倡导的自由民主信条与实际黑 人受到的不公正待遇二者之间的巨大反差; 第二次世 界大战令更多人认识到了种族主义的危害; 联邦政府 为了争取非裔美国人支持所做出的有利政策与全国 有色人种促进协会的努力与自由主义宪政革命。以上 几点原因便是促成五六十年代民权运动的主要原因。

\section{1. 长期的蓄奴制经济为种族隔离提供了土 壤}

自从黑人踏上美洲大陆的时候, 他们就失去了作
为人的资格, 成为了奴隶, 并且主要集中在美国南部, 为种植园主奉献劳力。依靠着“本质主义”的理论支持, 黑奴被视为一个在道德与治理层面的双重低下的种 族。虽然北部各州比南部更早废除奴隶制, 但南部由 于劳动力短缺, 所以仍然保持着落后的蓄奴制经济, 奴隶主甚至以罗马帝国作为为奴隶制开脱的理由 (罗 马帝国实行奴隶制度, 其超过百分之二十的人口是奴 隶)。在林肯正式颁布《解放黑人奴隶宣言》之后, 许 多奴隶已由林肯在恢复自由身, 但他们战后的地位仍 然不明。美国联邦参议院于 1865 年 1 月 31 日批准了 美利坚合众国宪法第十三修正案, 以废除奴隶制和强 制劳役, 但诸如黑人法令、白人至上主义等等因素依 然为非裔美国人族裔带来极其沉重的灾难。

美国在经历南北战争之后, 开始着手于重建美利 坚合众国的国家秩序，这一时期被称为“重建时期”

(Reconstruction), 学界传统上将其划定为内战后期 或者战争结束后北方联邦政府为使南方各州重新加 入合众国所实施的军事管制时期，时间上大致划在 1863-1877 年之间。但在司法重建方面, 美籍华人 学者王希教授和美国宪政史学家帕梅拉·布兰德温教 授等学者则认为在 1899 年时联邦最高法院认为“隔离 但平等”原则之后为司法重建的完成。1 870 年左右, 南方各州已经基本回归联邦, 同时在 1873 年经济危 机后，政府已经无力调配资金以对南方进行军事管控。 随着非裔美国人争取公民权的运动愈发强烈, 白人亦 日益感受到某种程度上的危机, 使大量共和党国会议 员们加入民主党。同时, 此时在共和党内部又掀起了 一股主张妥协的浪潮, 一改共和党重建初期的政治价 值取向。可以发现, 连最高法院对于种族问题依然选 择冷漠处理, 其他的政治机构与社会也基本采取了类 似的态度。

\section{2. 种族隔离导致黑人族裔与白人族裔的分 裂}

虽然《解放黑人奴隶宣言》与美利坚合众国宪法 的颁布与通过帮助非裔美国人摆脱了奴隶的身份, 但 这一群体仍旧受到种族隔离的迫害, 特别是在重建时 期之后的美国南方。种族隔离指的是指在日常生活中, 按照不同种族将人群分割开来, 使得各种族不能同时 使用公共空间或者服务。在美国, 黑人无法居住在城 镇较为发达的地方, 只能被迫一同蜗居在环境奇差的 地方, 人口密度大, 住宿条件非常差。学校和教堂也 实行种族隔离, 甚至连餐厅百货大楼都无法使用。黑 人无法与白人一同使用公共设施。同时, 黑人在纳税 的情况下，却往往无法获得投票权。同时在工作与经 济方面, 由于工业革命的发展与农业向需求更少劳动 力的方向发展，导致大量的非裔美国人从南方州来到 北方寻找工作，但由于种族隔离，使得这些从南方迁 移过来的移民经济地位非常低下, 难以享受到跟白人 一样的福利与保障。同时非裔美国人更多的是作为一 种白人的替代品出现, 当白人进行罢工与游行示威时, 非裔美国人就会来填补工作的空缺，进而引发了黑人 
与白人群体的不断对立, 加大了两群体的冲突。

\section{3. 强种族主义倾向与平等信条的冲突}

亨廷顿于《美国政治一激荡于理想与现实之间》 一书中提出了“美利坚信条”的概念，其指的是自由、 平等、民主、宪政、个人主义，由《独立宣言》最先 阐明, 但其渊源却可以追溯到启蒙运动时期的洛克式 自由主义以及新教教义中的千禧年主义和道德主义。 当清教徒在弗吉尼亚北部登陆的时候, 这一批为了逃 离宗教压迫，为了反抗权威、抵制特权和寻求民主的 人们, 就此签订下了美国历史上第一份重要的政治文 献, 即《五月花公约》, 他们放弃了传统的君主制度, 而是采用了让渡各自一部分的权利, 以契约的形式组 建“利维坦”, 清教主义也就此成为了美利坚信条

(American Creed) 的基础。平等也是美利坚信条中 非常重要的一部分，其影响了一代又一代的美国公民。 但黑人始终无法享受平等, 被迫忍受种族隔离与身份 歧视。美利坚自建国以来所倡导的人人平等与自由的 信条，与彼时存在的强种族主义倾向形成了巨大的反 差, 不断地折磨着非裔美国人的内心, 也激起了大量 非裔美国人的反抗心理。马丁.路德·金的父亲曾经前 往一个鞋店, 当时他的父亲拒绝走到后面的房间等待 服务, 他如是写道: “这是我第一次见到父亲如此怒不 可遏......那次经历使我很早就明白我的父亲并没有 适应这种制度 (种族隔离), 他在帮我树立正确的是 非观念方面起到了重要的作用。我依然记得, 我和父 亲并肩走在街道上时, 他嘴里喃喃自语道: ‘这种制度 我不在乎还要忍受多久, 但我绝对不会接受。”大部 分的美国人已经无法忍受种族隔离带来的困扰, 在长 期的情感积蓄之后终于爆发, 演变成轰轰烈烈的运权 运动。

\section{4. 第二次世界大战令越来越多人认识到种 族主义的危害}

第二次世界大战将不同的种族, 不同的利益群体 都置于共同的目的之下, 也就是反对纳粹, 击败轴心 国, 赢得第二次世界大战。这一点使得美国开始意识 到团结全国的重要性以及种族问题带来的危害。罗斯 福总统在第二次世界大战期间提出了 “四大自由

(Four Freedoms)”, 也就是言论自由（freedom of speech and expression), 宗教自由（worship God in his own way)，免于贵乏的自由（freedom from want）与 免于恐惧的自由（freedom from fear），这“四大自由” 成为了对纳粹进行战争的口号。在二战时期想要要求 美国参加欧洲战争的美国人也将自己称作“为自由而 战的委员会”（Fight for Freedom Committee）。他们坚 信纳粹的毁灭是对第一修正案所授予的自由的最大 保护。可见自由已经成为了美国参加二战的最强有力 的口号。二十世纪三十年代, 美国的左派提出所有的 美国人应当享受所有自由所带来的福利。而这在二战 中也成为了罗斯福政府的官方态度与立场, 罗斯福政 府也大幅动用大众媒体以宣传非裔美国人在美国历
史中做出的贡献，以促进各种族之间平等相处。其中 一个电台节目就如此问到听众: “如果我们继续为其 他族裔设立障碍, 那我们如何打赢这场战争? 而胜利 的原因正是这伟大的国家致力于倡导人人生而平等。” 这让大量的美国人认识到种族主义所带来的弊端, 也 开始对长期遭受种族隔离与种族歧视的非裔美国人 群体施以关注。而纳粹主义令世界深刻认识到了种族 主义的危害。因此, 尽管美国种族歧视观念根深蒂固, 但在希特勒的种族主义受到谴责下, 美国对非裔美国 人的种族歧视与种族隔离政策, 也得不到全世界的支 持, 美国种族主义根基开始动摇。非裔美国人非常支 持所谓的“民主战争”（war to make the world safe for democracy）抗击轴心国，但最后不论是这种“自由”意 识形态还是他们在战争中的贡献都没能为他们带来 在公民权利方面的改善。使得大量非裔美国人认为改 善国内的民主状况的重要性高于击败纳粹的重要性。 有一位老兵如此说道: “我们在海外为民主而战斗, 我 认为我们理应在国内也应当享受一定的民主”。同时， 在海外奋战的黑人士兵, 也体会到了在美国国内从未 体会到的自由与良好地对待。如此也促使越来越多非 裔美国人开始为自己族裔的权利开始奋斗。

\section{5. 联邦政府出台保护非裔美国人的政策}

如果将民权运动仅仅看成是黑人运动, 就无法解 释为什么在二十世纪五六十年代达到高潮。只是如果 把联邦政府也纳入考量范围, 便可以解释这一问题。 自罗斯福总统所进行的一些社会救济措施与公共工 程计划帮助非裔美国人群体在经济上得到了一定的 福利, 这带给了非裔美国人群体争取自身权利的希望。 二战之后, 在杜鲁门政府时期, 黑人领袖希望杜鲁门 对美国社会中的种族歧视和隔离采取行动。非裔美国 人利用美国在二战中不断宣传民主的背景下，以民主 来迫使美国政府着手处理国内的种族问题，并且推行 维护和保障非裔美国人的权利的政策。同时在二十世 纪中叶, 非裔美国人的政治影响力逐步加大。加之自 一战以后大量的南部黑人向城市移居, 加强了北方非 裔美国人群体的政治力量，至杜鲁门政府时期，非裔 美国人群体的政治影响力已经不可小䖋。而且非裔美 国人群体已经不再能容忍种族隔离带来的生理与心 理上的压迫。在罗斯福总统当政时期, 非裔美国人就 以游行为威胁, 要求政府实现各族裔在工作上的平等, 罗斯福总统被迫推出 8802 号行政命令来解决这一问 题。可见非裔美国人群体日益强大的政治力量。在此 基础上,1946 年 12 月 5 日杜鲁门发表总统声明宣称:

“我国比世界上任何其他地方更充分地认识到了免于 恐惧的自由。然而，我们当中并不是所有人都平等地 免于恐惧之苦。在某些地方, 这种免于恐惧的自由还 不时地受到严重的威胁。在战争结束后, 情况就是这 样的。因为种族和宗教信仰的原因, 有组织的群体一 直在煸动仇恨和不宽容的思想。不时有暴徒通过暴力 行动把恐惧注入人们的心中。”并在随后创建总统民 权委员会, 专门负责调查研究民权保障问题, 最后该 委员会出台报告, 即《保护这些权利》, 并且提出了保 
护非裔美国人权利的具体措施。由于杜鲁门在保护非 裔美国人权利方面功绩卓著, 收获了大量黑人群体的 支持。比如 1948 年杜鲁门总统就依靠城市黑人群体 的支持平衡了被南部各州的民主党党员（Dixiecrats） 夺走的选票。而杜鲁门对于黑人权利的保护与支持也 给予了黑人群体更多的希望, 鼓励他们进行更多尝试 实现平等, 进一步将民权运动推向了高潮, 达到了五 六十年代的民权运动高峰。

\section{6. 全国有色人种促进协会的努力与自由主 义宪政革命}

全国有色人种促进协会 (National Association for the Advancement of Colored People）是美国的一个非 裔美国人民权组织, 始于 1909 年 。自上世纪五十年 代开始, 该协会认为消灭种族歧视的时机已经来临。 大量的非裔美国人相信所谓“双重胜利” (double V for victory), 即二战胜利不仅带来了同盟国对轴心国的 胜利, 同样也为种族隔离的消除带来了曙光。同时朝 鲜战争时期, 黑人士兵与白人士兵的同心协力, 也证 明了两大族群是可以相互合作的。在此基础上，1954 年, NAACP 向法庭提起诉讼, 挑战学校的种族隔离, 并且最终胜利, 也就是著名的布朗诉托皮卡教育局案

(Brown v. Board of Education of Topeka), 这一判决 带来了美国种族隔离在宪政方面的基础崩裂，是在 民权问题上的一次巨大革命。同时, 时任美国首席大 法官的厄尔.沃伦也做出了不可磨灭的贡献, 其主政 的法院作出了一系列里程碑式的判决, 除去布朗诉托 皮卡教育局案之外, 还有 1964 年的亚特兰大之心汽 车旅馆诉美国案 (Heart of Atlanta Motel, Inc. v. United States）以及 1967 年的洛文诉弗吉尼亚州案（Loving $\mathrm{v}$. Virginia)，这些判决严重动摇了种族隔离制度的基 础, 给予非裔美国人族群以获得平权与自由的希望, 推动了自由主义宪政革命。比如当时的黑人民权运动 领袖之一, 罗伯特·威廉在了解到布朗诉托皮卡教育 局案的判决之后, 就表达了极度的喜悦, 他表示这种 喜悦就好似将近一个世纪以前, 在南北战争中的黑奴 听闻到《解放黑人奴隶宣言》时一样。

\section{7. 象征性带来矛盾的激化}

在这一时期的民权运动当中, 象征性一直是一个 普遍的问题。虽然马丁路德金的演讲振奋人心，但也 有着致命的问题，过于空泛而没有什么实质性的措施。 而在美国真正开始改革的时候, 也存在这个问题。比 如在布朗案之后, 部分温和白人群体进行的象征性改 革。该群体认为在学校问题上, 象征性的改革一也 就是让黑白种族合校是具有一定的积极意义的, 由于 非裔美国人在经济尚不能与白人相匹敌, 所以一般只 有少量黑人学生可以与白人学生一同学习, 也可以让 白人种族在这个过程中逐步接受非裔美国人。而南部 的温和派认为, 这一切需要缓慢的改革, 不能激起反 对派白人的激烈反抗。虽然这一白人群体具有一定的 进步意义, 但是图讨好黑白两大群体的妥协性改革不
仅不能使任何一方满意, 而且还激化了大量群体的矛 盾。对于非裔美国人族群来说。统计数字显示, 在 1960 年 12 月、1964 年 5 月和 1965 年 6 月, 北卡 罗来纳州入读白人学校的黑人孩子分别是 $82 、 1865$ 和 4963 人, 只占黑人孩子的 $0.02 \% 、 0.537 \%$ 和 $1.42 \%$ 。 改革力度非常不够, 需要进一步实施更加激进的改革。 同时白人群体认为该改革已经过于激进, 黑人在文化 层面依然不具有与白人一同学习的能力。可见, 象征 性的改革进一步激化了黑白两大群体的社会矛盾, 这 也使得非裔美国人群体的行为更加激进, 最终导致了 1960 年的“入座运动”的爆发。越战的爆发于马丁路德 金的被谋杀, 加之美国社会深刻的经济衰退, 其中所 带来的绝望的感觉促使越来越多的非裔美国人采取 更加激烈的运动, 为 1965 年以后逐步停止的运动注 入了新的活力。

\section{4. 二十世纪五十至六十年代民权运动带来 的影响}

这场运权运动, 在普遍的评价上都是积极的, 在 教育，住房，政治权利等方面改善了非裔美国人的处 境, 使这一群体逐步与白人群体走向平等, 虽然仍有 部分领域非裔美国人仍然遭受着不平等, 美国社会也 存在一定的种族歧视问题, 但这次运动带来的积极影 响依然不可磨灭。除此之外, 这次运动也带来了消极 影响, 一定程度上撕裂了美国自由主义与保守主义的 平衡状态。

\section{1. 白人族裔与黑人族裔的逐步和解}

自民权运动之后，全美各地对非裔美国人的态度 都得到了非常大的缓解, 尤其是在南部地区。根据 1972 年，1976，1980 年与 1984 年所进行的“一般社 会调查” (General Social Surveys), 法尔博教授

(Firebaugh) 与戴维斯教授 (Davis) 展示出了强大的 证据以证明南部白人的“种族傲慢”（Racial Prejudice） 态度下降程度要快于北方 (南部对非裔美国人的态度 自始至终都要比北方更加恶劣)。可见民权运动对美 国社会冲击之深。南方各州的种族态度逐渐与北方各 州没有多大差异。虽说目前在南部各州已经出现了所 谓“新南方”思潮, 即重新对黑人抱有敌对态度, 但相 比民权运动之前，其烈度已经下降。

\section{2. 非裔美国人权利的逐步平等化}

同时非裔美国人也在此次运动中基本实现了其 目的。在社会认识方面，越来越多的白人在民权运动 之后对于非裔美国人表达出了充分的宽容与对种族 融合的广泛接受。在教育方面, 民权运动后到 1994 年 黑人的高中毕业率提高了 3-4 倍, 和白人的差距也大 大减小。同样,在大学教育方面, 黑人也取得了很大的 进步。1 940 年, 年满 25 岁以上完成大学教育的黑人 人数在其总人口中的比例是 $1.5 \%$, 而 1993 年这个数 字达 $13 \%$, 而黑人女子的大学毕业率高于黑人男子两 
倍。在投票权方面, 面对愈演愈烈的抗议冲突, 美国 国会于 1964 年通过了民权法案 (The Civil Rights Act), 于 1965 年通过了投票权法案 (The Voting Rights Act), 这两份法案充分保障了非裔美国人的投票权, 在此之 前，某些州会使用“投票税”（Poll Tax）或者“识字测 试”（Literacy Test）来限制非裔美国人行使自己的投 票权。再次之后非裔美国人投票率越来越高, 在 80 年 代中期已经达到了百分之六十六, 基本达到了白人族 裔的投票率水平。至 1992 年, 已经有超过 8000 千名 非裔美国人拥有了政治职位。

除去民权法案与投票权法案, 美国国会在 19651972 年先后推出了“平等居住法案” (The Fair Housing Act）与平等机会法案 (The Equal Opportunity Act), 保证了非裔美国人在住房方面与就业方面免受歧视 与不公正对待。

由此可见, 民权运动在社会层面最大程度消减了 白人族裔对非裔美国人族裔的歧视, 逐步消解了两方 的对立情绪, 虽然并不完全, 但依然是一次巨大的进 步。在立法方面的成就为黑人在教育和就业方面提供 了法律保障，投票权使得黑人可以更好地参与政治 活动, 这些参与政治的非裔美国人也会在国会维护非 裔美国人群体的利益。

\section{3. 仍然存在的种族歧视问题}

虽然自民权运动之后, 所谓的种族隔离已经在法 律上被确认为违法, 但在民间依然存在着针对于非裔 美国人的种族歧视问题。在 2020 年就发生多起针对 于黑人的暴力事件, 多次出现了白人警察杀害黑人的 恶性事件。自新冠肺炎爆发以来, 根据美国国家公共 电台 (National Public Radio) 新闻所报道, 至 2020 年 四月, 纽约州因新冠肺炎病死的病人中, 非裔美国人 与拉丁裔美国人病死的概率是白人的三倍, 可见美国 社会依然存在严重的种族问题。同时, 美国人对待这 一种族歧视也抱有一种越来越冷漠的态度。根据调查 发现, 只有约三分之一的被调查人认为弗洛伊德事件 可以给美国社会带来巨大的改变, 剩下绝大部分人认 为这一事件不会带来多少冲击与改变。大部分美国人 知识学习到了之前一些关于种族主义和种族隔离的 历史, 并不会将他们引申到现代。由此可见爆发于上 世纪的民权运动的的确确带来了改善, 但依然存在余 䔣。同时, 在经济平等问题上, 非裔美国人相对于白 人族裔来说, 依然处于严重的不利地位, 大量的非裔 美国人生活在贫困之中。可见, 民权运动并不彻底, 而且民权运动所带来的改善是给予了非裔美国人作 为美国公民的合法权利, 而忽略经济层面上的贫富差 距问题, 也昭示着这项运动本身并不非常彻底。

\section{4. 加剧了美国社会的撕裂}

自 1968 年以来的社会大变迁，导致了“道德”的 分化, (本质上带来了建制派与非建制派的冲突, 自 由主义 (或者也叫新自由主义) 者与保守主义者之间
的冲突，本文主要聚焦于后者进行论述）保守主义者 与自由主义者所认为的道德王国并不一致，自由主义 倾向于实现人人平等的团结美利坚, 关怀少数族裔和 弱势群体，比如捍卫黑人权利与 LGBTQ。而保守主 义根植于个人自由。简单来说, 自由主义倡导人人平 等, 人人皆有自由, 保守主义则倡导自由至上, 强调 个人权利。但在 1968 年后, 民权运动逐渐将身份政 治从所属的亚文化推向主流文化的范畴, 与中下层 “定居者”白人群体的生活失意与多元文化入侵形成 鲜明对比, 自由主义与保守主义之间的和平状态彻底 撕裂。两方之间所属的道德范畴完全不同, 而自由则 给予了两方捍卫权利的能力, 两方都视对方的政治主 张损害了自己的利益, 造成了美国自由主义者与保守 主义者的严重对抗，在经济全球化加大社会分化的影 响下, 进一步直接撕裂了美国社会。针对于种族歧视 问题, 在 2017 年有百分之六十四的民主党人士认为 黑人的困境是由于种族歧视导致的，但只有百分之十 四的共和党人士持有相同观点。

同时，民权运动之后，所谓的“平权”开始走向了 极端化, 政治正确愈发偏离了原本的意义。比如凡是 带“黑”的、“有色”的词汇，统统成了禁忌。“黑名单”

（blacklist）变成“blocklist”，甚至连游戏公司也对游 戏内存在的所谓“种族歧视”言论大加管控, 而忽视了 其他关键问题。同时，非裔美国人在政治上实力也不 可小觑, 所以也有越来越多的政客为了赚取非裔美国 人群体的选票，进一步推行过度的政治正确讨取欢心， 进一步恶化政治正确所带来的影响, 将迫使美国政治 体制向“侍从型”发展, 完全为选票服务, 加速美国政 治衰败。美国社会被套上了“政治正确”的牢笼，大量 反对的声音都会被曲解为法西斯主义与右翼言论, 反 而限制了言论自由，消解了美利坚信条所强调的“自 由民主平等的”的积极意义。政治正确如同一把双刃 剑, 不仅保护了少数族裔和弱势群体, 也不可避免的 导致了极端的言论环境，使得恐惧蔓延，交流效率低 下。此外, 在 2020 年五月爆发的弗洛伊德事件, 也 反映出反种族歧视越来越向极端化发展, 以反种族歧 视为名的暴力示威冲突越来越多, 去年美国就完全笼 罩在新冠肺炎与暴力示威的阴影之下, 让人对美国一 直以来所标榜的“理想之地”产生巨大的怀疑。无数的 非裔美国人走上街头, 甚至还有大量的不法之徒趁乱 打砸抢, 如此造成了难以想象的混乱秩序。而这却与 托克维尔所论述的好似理想之地德美国形成了鲜明 的对比。从中可以窥见非裔美国人更多地是注重于他 们的肤色而并非是美国人的政治身份，将困境的根源 完全归结为美国制度的原因。在白人族裔层面, 在特 朗普政府政策的推动与弗洛伊德事件之后，他们对于 种族歧视问题的认识也产生了巨大分化，他们有的人 走上街头与非裔美国人一同抗议, 也有人愈加憎恨非 裔美国人，白人与黑人的分歧也越来越大。 


\section{大多数黑人成年人对国家种族进步持负面看法}

美国的种族关系普遍不好

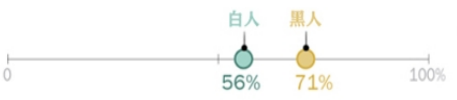

特朗普让种族关系恶化

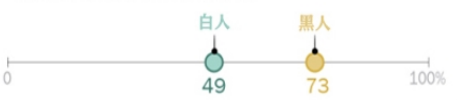

奴隶制的残留在很大程度上影响了黑 人在当今美国社会中的地位

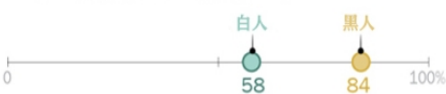

\section{我们国家在给予黑人与白人平等的权} 力方面做的还不够

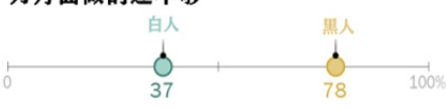

黑人最终不太可能获得平等的权力

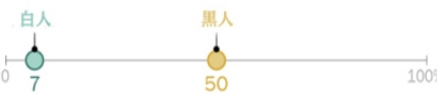

图 1 黑人成年人对国家种族进步的看法

数据来源: (Race in America 2019, https://www. pewresearch. org/socialtrends/2019/04/09/race-in-america-2019/,

Accessed 25 May. 2021)

上图为一项针对白人族裔与黑人族裔在看待种 族问题的调查，可以在其中可以清楚发现问题：黑人 族裔普遍认为, 当前美国社会的种族问题更加严重, 而白人则倾向拥有较为温和的态度, 不认为这一问题 已经到了无法容忍的地步。在最后一项“没有太多/完 全没有非裔美国人可以获得平等权利”这一项当中， 只有百分之七的白人赞同, 但黑人却有百分之五十持 赞同意见。在 2020 年美国大选结果出炉后, 虽然拜 登获得了最终的胜利, 但特朗普依然获得了比 2016 年更多的票数, 虽然已经进入拜登政府时期, 但这不 意味着对特朗普主义的彻底抛弃, 所谓的“治愈伤口”, 越发遥不可及。

\section{5. 弥合社会危机}

综上所述, 可以发现美国深陷身份政治危机, 政 治正确成为了一把利剑而不是保护少数族裔的盾牌。 非洲裔美国人为了自己的利益而肆意妄为。自由带上 了浓重的马基雅维利式的色彩, 以一言蔽之: “我的自 由才是自由”, 缺乏对于他人的理解与认同。究其原因, 首先过于混乱的运动, 其次便是过度激烈的左派思潮。 面对社会的分裂与危机, 美国必须做出改变。针对这 一问题, 首先民权运动本身入手。在上世纪六十年代, 马丁路德金已经逐步探索出一条合适的民权运动之
路, 各种恰当的组织与纲领也在孕育之中, 但是马丁 路德金在三十九岁时便被暗杀, 整个民权运动朝着无 序与混乱发展。目前的运动当中, 缺乏领导与纪律, 才出现了如上文所言的打砸抢的混乱事件, 进一步激 化了非裔美国人与白人之间的对立情绪。所以黑人需 要发展自己的政治团体, 将整个运动推向有序化, 真 正明确非裔美国人为何处于困境, 明确需要什么权利 才可以改善当前的困境。在众多思潮中，黑人爱国者

（black nationalists）认为民权运动是个幌子, 要实现 平权就要发展出自己的一套自治的经济, 社会与文化 建制, 这一观点相对比较正确, 黑人民粹主义者 (black populists）也提出了要复兴诞生于民权运动之中的行 动者黑人政治文化 (activist black political culture)。如 果按照此种方式进行下去, 相信混乱与无序可以很快 消失在民权运动当中。同时可以从言论管控方面入手, 通过各个族裔, 群体的更多良性接触, 创造各群体之 间的理解。虽然美国一直拥有言论自由的传统，但在 面对前任总统特朗普不断使用推特煽动民粹暴力的 情况下, 美国也已经开始对于言论自由进行改造。福 山已经提出可以创造操作系统与应用之间的所谓“中 间件”(middleware)公司的竞争层, 也就是如 Facebook 这样的公司将内容审核的任务外包给这些中间件公 司, 削弱大平台的力量, 使得边缘声音和小众声音可 以被倾听到，改变“政治正确”一言堂的局面。同时 Facebook 也设立了言论检查委员会来对暴力性言论 进行控制，相应的也可以促进非裔美国人与其他族裔 的对话, 由此建立共同信任，逐步消解政治正确带来 的恶性影响。

\section{5. 结论}

每一个民族都应当享有平等的权力, 而不因为他 们的肤色就获得歧视和不公正的待遇。“人皆生而平 等”, 是美国独立战争时期使用的名言, 信条的内涵当 中也包括了自由平等。所以不可否认，上个世纪，非 裔美国人群体为了追求美利坚平等权利做出的一切 努力都应当得到积极的认可。他们的努力也为后世的 非裔美国人创造了更好的生活环境与条件，而不用忍 受屈辱。虽然目前依然存在不少的种族歧视现象, 比 如白人警察的暴力执法，在弗洛伊德之后，又出现了 多名非裔美国人遭受警察暴力致死，非裔美国人失意 的生活状况依旧昭示着种族问题的严重。但相比于上 个世纪民权运动之前，已经有了十足的进步。虽然民 权运动值得袈奖, 但这一运动更多地是赋予非裔美国 人以作为“人”的合理权利，在平等问题上，尤其是经 济平等问题上始终与理想状态存在鸿沟。在所谓“美 国梦”的定义中，美国赋予每个美利坚公民以相同的 人生起点与权利, 但实现人生价值便依赖于自己, 简 而言之经济问题是属于个人的。美国的制度缺陷与巨 大的收入分配差距加剧了政治极化，美国的基尼系数 从 1970 年开始直线殺升, 在 2000 年就已经达到了 0.462 , 越来越接近在大萧条时期的 0.57 的危险水平, 同时收入隔离问题也越来越严重, 种族之间的收入愈 发不平等。 


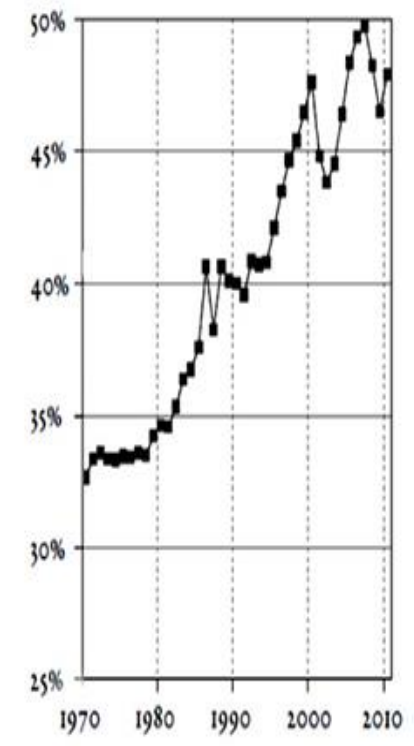

\section{收入前10\%人群的收入占国民收入的比重}

\section{图 2 收入前 $10 \%$ 人群占国民收入的比重}

（数据来源：林红. “达尔之问” 的再讨论: 经济不 平等与美国的再分配困境 [J]. 美国研

$$
\text { 究, 2021, 35 (02) : 9-30+5. ) }
$$

虽然美国曾经实施过凯恩斯主义经济学，但在陷 入上世纪七十年代的滞涨之后, 开始重新回归自由主 义经济学, 推行减税政策, 刺激企业活力, 但却导致 美国政府在税收问题上疲软, 无法筹集足够的公共资 金以改善美国社会存在的严重的经济不平等现象。由 此反映出实现彻底的种族平等依然有很长的路需要 走。同时, 后世非裔美国人族裔将所谓平权推向特权, 非裔美国人愈发试图将自身所处的亚文化范畴推向 主流范围, 追求过度的政治正确, 势必会激起社会的 强烈反抗与不合，过度“政治正确”目前所带来的更多 的是消极的, 在黑人与白人经济收入差距过大的前提 下进一步将美国推入 “身份政治”困境, 导致美国无法 凝聚全国力量形成“共同身份”（shared identity）。“反 种族歧视”与“平权运动”也就成为失意的非裔美国人 发泄怒火的工具, 而丧失了原本的含义, 他们将一切 都归各于其他族裔带来的压迫, 而忽略了造成他们困 境的自身原因, 所以非裔美国人需要建立自己的组织, 使得整个运动更加的合理而有序, 而不是任由自己的 怒火随意发泄。这样只会越来越激化矛盾。

\section{REFERENCES}

[1] Burson, George. "The Black Civil Rights Movement.' OAH Magazine of History, vol. 2, no. 1986, pp. 35-40. JSTOR, www.jstor.org/stable/25162501. Accessed 24 Mar. 2021.

[2] Gaines, Kevin. “The Civil Rights Movement in World
Perspective." OAH Magazine of History, vol. 21, no. 1, 2007, pp. 57-64. JSTOR, www.jstor.org/stable/25162103. Accessed 27 Mar. 2021.

[3] Eric Foner. "The Contested History of American Freedom.” The Pennsylvania Magazine of History and Biography, vol. 137, no. 1, 2013, pp. 13-31. JSTOR,

www.jstor.org/stable/10.5215/pennmaghistbio.137. 1.0013. Accessed 13 Apr. 2021.

[4] Ulmer, S. Sidney. "Earl Warren and the Brown Decision." The Journal of Politics, vol. 33, no. 3, 1971, pp. 689-702. JSTOR, www.jstor.org/stable/2128278. Accessed 14 Apr. 2021.

[5] Hillman, Arye L. "Political Economy and Political Correctness." Public Choice, vol. 96, no. 3/4, 1998, pp. 219-239. JSTOR, www.jstor.org/stable/30024391. Accessed 18 Apr. 2021.

[6] Washington, Robert. "Reclaiming the Civil Rights Movement." International Journal of Politics, Culture, and Society, vol. 9, no. 3, 1996, pp. 459473. JSTOR, www.jstor.org/stable/20019847. Accessed 19 Apr. 2021.

[7] Huntington, Samuel P. "American Ideals versus American Institutions." Political Science Quarterly, vol. 97, no. 1, 1982, pp. 1-37. JSTOR, www.jstor.org/stable/2149312. Accessed 1 Apr. 2021.

[8] Hall, Jacquelyn Dowd. "The Long Civil Rights Movement and the Political Uses of the Past." The Journal of American History, vol. 91, no. 4, 2005, pp. 1233-1263. JSTOR, www.jstor.org/stable/3660172. Accessed 25 May.2021.

[9]Amid National Reckoning, Americans Divided on Whether Increased Focus on Race Will Lead to Major Policy

Change.https://www.pewresearch.org/socialtrends/2020/10/06/amid-national-reckoningamericans-divided-on-whether-increased-focus-onrace-will-lead-to-major-policy-change/.Accessed 25 May.2021.

[10] Stanford psychologist identifies seven factors that contribute to American racism. https://news.stanford.edu/2020/06/09/sevenfactors-contributing-american-racism/. Accessed 25 May.2021.

[11] The Civil Rights Movement in America. https://www.bbc.co.uk/bitesize/guides/zcpcwmn/re vision/1. Accessed 26 May.2021. 
[12] America Had a Reckoning on Race This Year. The Election Showed How Little Has Changed. https://time.com/5907733/race-in-america-2020election/. Accessed 27 May.2021

[13] Rethinking Political Correctness. https://hbr.org/2006/09/rethinking-politicalcorrectness. Accessed 1 June 1,.2021

[14] Langston, Donna. "Black Civil Rights, Feminism and Power." Race, Gender \& Class, vol. 5, no. 2, 1998, pp. 158-166. JSTOR, www.jstor.org/stable/41675328. Accessed 13 July 2021.

[15] BERGER, DAN. "Rescuing Civil Rights from Black Power." Journal for the Study of Radicalism, vol. 3, no. 1, 2009, pp. 1-27. JSTOR, www.jstor.org/stable/41887616. Accessed 13 July 2021. 